\title{
Neue Technologien, neue Versionen - neue Urheber? Fragen und Perspektiven zur Archivierung digitaler Inhalte am Beispiel der Elektroakustischen Musik
}

\author{
Miriam Akkermann
}

Künstlerische Arbeiten in musikalischen Bereichen wie beispielsweise der Elektroakustischen Musik und der Computermusik entstehen von jeher in enger wechselseitiger Beziehung mit aktuellen technologischen Entwicklungen. Geprägt in den 1950er Jahren in Frankreich, dient die Bezeichnung Elektroakustische Musik bzw. musique électroacoustique als eine Art Überbegriff für Musikstücke aus dem Bereich der klassischen (experimentellen) Musik, deren verbindende stilistische Merkmale sich weitgehend aus der Verwendung elektroakustischer, elektronischer und digitaler Klangerzeugung oder Klangveränderung konstituieren. ${ }^{1}$ Computermusik wird in der Regel der Elektroakustischen Musik zugeordnet und im weitesten Sinne als eine Musik definiert, für deren Entstehung die Verwendung eines Computers wesentlich ist. Computer werden dabei sowohl zur (digitalen) Klangerzeugung (Klangsynthese) als auch zum Errechnen von Notation (Partitursynthese) verwendet. ${ }^{2}$

Die schnellen Entwicklungen gerade im Bereich digitaler Technologien führen zu raschen Generationenwechseln bei Hard- und Software, was zu neuen Herausforderungen führt: In der Mixed Music - Musik, die elektronische und analoge Elemente enthält ${ }^{3}$ ist beispielsweise bei Kompositionen aus den 1980er und 1990er Jahren zu beobachten, dass die bei der Premiere verwendeten Technologien heute, zu Beginn des 21. Jahrhunderts, oft veraltet und manchmal nicht mehr funktionstüchtig sind. Für neue

* Prof. Dr. Miriam Akkermann ist Juniorprofessorin für empirische Musikwissenschaften an der TU Dresden. Ihre Forschungsschwerpunkte sind Elektroakustische Musik, Computermusik und Musiktechnologie, Archivierung von musikalischen Arbeiten, sowie Aufführungspraktiken und ihre Herausbildung.

1 Vgl. Supper / Ungeheuer, Art. Elektroakustische Musik, https://www.mgg-online.co $\mathrm{m} / \mathrm{mgg} /$ stable/11329 (zuletzt abgerufen am 15.5.2020).

2 Vgl. Supper, Elektroakustische Musik und Computermusik, 26, sowie ders., Art. Computermusik, https://www.mgg-online.com/mgg/stable/12446 (zuletzt abgerufen am 15.5.2020).

3 Vgl. Tiffon, Espace et musique mixte, sowie Supper, Elektroakustische Musik, 26. 
Aufführungen bedeutet dies z.B., dass ursprüngliche Technologien mit neueren (aktuelleren) Geräten inkompatibel sind oder der originale Code, bedingt durch eine enge Hardware-Bindung, nicht mehr direkt auf neuen Rechnern ausgeführt werden kann. Gleichzeitig ist die Dokumentation der ersten Aufführungen, entgegen ihres jungen Alters, teilweise lückenhaft. In der Folge müssen für neue Aufführungen in der Regel neue Versionen des technischen Set-ups bzw. neue Fassungen des (Programm-)Codes erstellt werden. In der Betrachtung ist nun zu klären, wie diese neuen Versionen einzuordnen sind, also ob es sich um Interpretationen der ursprünglichen Kompositionen oder neue Fassungen handelt und in welchem Verhältnis diese zu den ersten Quellen stehen.

Der Moment der notwendigen Erneuerung eines vorhandenen Codes scheint also ein Tipping Point zu sein, der von einer Spirale aus (digitalen) Entwicklungen und den daraus erwachsenden, immer neuen Versionen, die sich zur Erstfassung einer Komposition gesellen, angetrieben wird, und der in Bezug auf das Urheberrecht zu zwei zentralen Fragen führt: Wer ist als Urheber:in der immer neuen (Code-)Versionen zu sehen? Und was bedeutet das jeweils für die Nutzungsrechte?

Aus technischer Sicht ist eine konstante Notwendigkeit gegeben, die vorhandenen Codes zu erneuern oder zumindest anzupassen. Die daraus resultierenden, immer neuen Codes werden als neue Versionen abgespeichert und gesellen sich im Sinne der Quellenlage zu der Erstfassung einer Komposition. Wer kann, soll oder muss nun als Urheber:in der neuen Codes angesehen werden? Dies ist nicht nur eine ideelle Frage nach dem künstlerischen Beitrag der Ersteller:innen, sondern hat direkte Folgen für die (Nach-)Nutzungsmöglichkeiten, z.B. die Archivierung und vor allem für die Zugänglichkeit zu diesem Material. Denn je nachdem, wie die Frage nach den (Urheber-)Rechten geklärt wird, ist es in einigen Fällen überhaupt erst möglich, Codes neu umzuschreiben, anzupassen oder zu aktualisieren und diese (neuen) Daten dann auch wieder für Aufführungen, Forschung oder Archivierung zur Verfügung zu stellen. Dies beeinflusst gleichzeitig auch die generelle Aufführbarkeit eines Stücks. Denn: werden die Codes nicht gepflegt, so werden sie über kurz oder lang unausführbar und die Kompositionen damit nicht mehr aufführbar. ${ }^{4}$ Die Möglichkeit einer technischen Anpassung ist somit für die adäquate Archivierung einiger Arbeiten zwingend nötig, da dies den Zugang zu den Inhalten sichert;

4 Eine Darstellung zu dieser Problemstellung und ihren praktischen Auswirkungen, sowie Überlegungen, wie diesen Herausforderungen begegnet werden kann, bietet u.a. Sebastian Berweck in seiner Dissertation (Vgl. Berweck, It worked yesterday). 
dass dies unter Umständen mit einer Klärung der Nachnutzung in Verbindung steht, wird bisher - jedenfalls im Bereich der Archivierung von Computermusik - kaum thematisiert.

Diese Mechanismen sind nicht nur in der Elektroakustischen Musik und der Computermusik, sondern auch in anderen künstlerischen Bereichen wie beispielsweise der Medien- oder Netzkunst zu finden, bei denen (digitale) Technologien einen substantiellen Bestandteil der Arbeit darstellen. Die Herausforderungen sind dabei nicht auf die technische Pflege der Inhalte beschränkt, sondern betreffen auch inhaltliche Aspekte, so sind unter Umständen neue (digitale) Archivstrukturen nötig, um die archivierten Informationen adäquat darstellen und unabhängig der technologischen Situation (dauerhaft) auslesen zu können. Die zugehörigen Debatten, Ansätze und Lösungsstrategien hierzu sind ebenso jung wie die dabei eingesetzten Technologien. ${ }^{5}$

\section{Neue Technologien - neue Codes: Herausforderungen am Beispiel der Mixed Music}

Bedingt durch ihre künstlerische Anlage zeigt sich in der Mixed Music eine Quellenlage, die für die genannten Herausforderungen sehr gut exemplarisch betrachtet werden kann: Die Kompositionen beinhalten sowohl Informationen für involvierte Musiker:innen, z.B. Notation oder Spielanweisungen, sowie einen elektronischen Teil, der von Audiodateien (vorgefertigte Tonspuren, die oft als ,Tape ${ }^{6}$ bezeichnet werden) bis hin zu Programmcodes für Klangerzeugung oder -veränderung in Echtzeit reichen. Für die Aufführung der Stücke ist die Nutzung von Technologien zwingend notwendig, sie unterliegen damit den grundlegenden Herausforderungen, die durch den technologischen Fortschritt entstehen. ${ }^{6}$ Zudem wird insbesondere für die Kompositionen der 1980er und 1990er Jahre zumeist eine Arbeitsteilung sichtbar. Am Institut de Recherche et Coordina-

5 Fragen danach, wie mit Versionengeschichte und nichtlinearen Anlagerungen von Inhalten umgegangen werden kann, werden nicht nur in der Musikwissenschaft diskutiert, sondern in allen kunstbezogenen Fachdisziplinen. Aktuelle Forschungsund Praxisansätze können beispielsweise auf der jährlichen Conference on Digital Libraries for Musicology sowie auf der Sonderveranstaltung ISEA Symposium Archives 2020 verfolgt werden. (Vgl. https://dl.acm.org/conference/dlfm und https://isea-arc hives.siggraph.org/, beide zuletzt abgerufen am 20.6.2020).

6 Weitere Ausführungen hierzu siehe u.a. in Akkermann, Vocabulary ruts in Mixed Music (unv. erh. b. Verf.). 
tion Acoustique/Musique IRCAM erarbeiteten Komponist:innen beispielsweise ihre Kompositionen mit Hilfe eines sogenannten Réalisateur en Informatique Musical RIM, welche:r sich in Absprache mit und unter mehr oder weniger Anleitung der Komponist:innen um die Programmierung und/oder Realisierung bzw. Handhabung der technischen Aspekte kümmerte. Die Komponist:innen mussten dabei nicht zwangsläufig Ahnung von den verwendeten Technologien haben, die RIMs erarbeiteten diese unter Umständen komplett eigenständig. Dies wurde - zumindest hinsichtlich der Urheberschaft des Stücks bei dessen erster Erarbeitung - nicht weiter hinterfragt. Die RIMs werden nicht bei Angaben zum Stück, jedoch zumeist bei der Premiere und ggf. nachfolgenden Aufführungen als solche mit angegeben. ${ }^{7}$

Werden nun neue Aufführungen erarbeitet, oder drohen Codes oder Tape aufgrund des technischen Fortschritts oder der physischen Vergänglichkeit ihres Trägermaterials verloren zu gehen, so entstehen neue Versionen der Inhalte, zumeist in digitaler Form und teilweise auch als reine Transfer-Versionen, die nur zum Sichern der Daten gedacht sind und auch nicht auf ihre Funktionalität getestet werden. ${ }^{8}$ Grundsätzlich gibt es verschiedene Ansätze, um neue Versionen zu erstellen, die unterschiedlich stark voneinander bzw. vom Originalcode abweichen. Grund dafür sind nicht nur technische Gegebenheiten oder der Zeitpunkt der Erstellung, sondern auch die Interessen der Erstellenden. So haben manche Versionen das Ziel eines möglichst Original-nahen Klangergebnisses, wofür auch ein weit abweichender Code akzeptiert wird, oder aber es soll ein möglichst Code-getreues Update verwendet werden, was mitunter jedoch ein sehr differierendes Klangergebnis verursachen kann. Wird die originale Programmiersprache beibehalten, so ist zumeist ein Update auf einen sehr ähnlichen Code bei sehr ähnlichem Klangergebnis möglich. Auch die Quelle, auf der eine neue Version fußt, kann einen Einfluss nehmen; wird beispielsweise auf reinen Transfer-Codes aufgebaut, so können ungewollte ,Übersetzungsfehler ${ }^{\varsigma}$ ob mangelnder Vergleichbarkeit mit dem Original weitergetragen werden. In einigen Fällen werden die programmierten Teile auch komplett neu erstellt, insbesondere dann, wenn es dazu strukturel-

7 Siehe hierzu beispielsweise die Angaben in der IRCAM Datenbank Brahms zu Kompositionen in der Kategorie ,electroacoustique' unter http://brahms.ircam.fr/w orks/genre/59/ (zuletzt abgerufen am 26.2.2020).

8 Diese Feststellungen basieren auf einer systematischen Betrachtung von Mixed Music Kompositionen, die von 1979-1991 am IRCAM erarbeitet und (erst-)aufgeführt wurden, sowie deren Wiederaufführungen im Zeitraum 2000-2010 und die zugehörigen Einträge in Sidney (vgl. Akkermann, Recherche 2016). 
le Beschreibungen in der Partitur oder der beigelegten Dokumentation gibt. Auch hierfür gibt es unterschiedliche Motivationen, die von Lehrformaten (Programmierübungen) über einen Wechsel der Programmiersprache bis hin zur Ermangelung anderer Programmcodes (veröffentlichte Partitur, jedoch kein Zugang zu den Patches) reichen. In der am IRCAM beheimateten internen Datenbank Sidney ist beispielsweise zu sehen, dass daraus ein Zuwachs an neuen Codes/Patches oder Versionen, die von unterschiedlichen Personen mit verschiedenen Zielen erstellt werden, resultiert. Zudem gibt es eine nicht benennbare Anzahl an weiteren, in Privatbesitz befindlichen Versionen, die im Rahmen von Aufführungen oder Tests auBerhalb des IRCAM entstehen. Es ist also anzunehmen, dass nicht alle Code-Versionen oder Set-up-Beschreibungen dem Quellmaterial einer Komposition dezidiert zugeführt werden, was zum Teil auch der Heterogenität in der Quellenlage geschuldet ist. ${ }^{9}$

In diesem Kontext stellt sich nun die Frage nach der Urheberschaft neu. Wer sollte als Urheber:in dieser neuen Codes und Patches gesehen werden, insbesondere wenn ein Programm völlig neu erstellt oder aus unvollständigen Quellen zu einem Stück rekonstruiert wird? Die Frage nach der ,Schöpfungshöhe' ist hierbei eher weniger zielführend, denn die Urheberschaft der Komponist:innen an der künstlerischen Arbeit, der originalen Komposition, ist nicht anzuzweifeln, auch wenn diese unter Umständen nicht alle Teile der Komposition selbst erarbeitet haben und auch wenn neue Aufführungen vom Original differieren. Dies wird, zumindest in den der ,klassischen Musik ${ }^{`}$ zugeordneten Bereichen, bei Abweichungen innerhalb eines genretypischen Akzeptanzbereichs in der Regel unter Fragen der Interpretation einer Komposition verhandelt. ${ }^{10}$

Wirft man jedoch einen Blick auf den durch diese Grundproblematik angelegten möglichen Freiraum, innerhalb dessen in der Mixed Music Codes neu erstellt werden können, so wäre es durchaus lohnend zu diskutieren, ob zum einen die RIMs nicht zumindest als Urheber:innen einzelner Teile einer Komposition gewertet werden sollten und zum anderen, ob heutige Computer Music Designer:innen und Computer Musicians nicht ebenso viel Schöpfungsanteil an den resultierenden Aufführungen haben wie die Person, die ursprünglich als Komponist:in anerkannt wurde.

9 Vgl. Akkermann, Recherche 2016.

10 Für den Bereich der Popularmusik ist dies abweichend, was sich beispielsweise an der Debatte zu ,Cover' und ,Sampling', sowie den damit einher gehenden rechtlichen Fragestellungen zeigt. Siehe hierzu u.a. Müllensiefen/Frieler, Beaucamp/Schrör, sowie Hondros in diesem Band. 
Die Annahme, dass sich Informationen im Laufe der Zeit ändern, je nachdem wie diese dokumentiert oder archiviert werden, ist aus vielen Musikgattungen und Epochen bekannt. So lässt sich gut nachvollziehen, dass Serge Lemouton und Samuel Goldszmidt hierbei von einem „cycle de vie des version“" sprechen, basierend auf dem sie den Dokumentationsprozess und die vernetzte Entstehung verschiedener Versionen der verfügbaren Quellen einer Komposition im Kontext der Datenbank Sidney formalisieren. ${ }^{11}$

Die hierbei erarbeitete Struktur basiert auf der grundlegenden Annahme, dass die Versionen als Teil des Originals im Sinne einer Versionengeschichte anzuerkennen sind. Die neuen Versionen des Codes werden also nicht als eigenständige neue Fassungen gewertet, unabhängig von deren technischer Ausgestaltung. Aufbauend auf diesen Überlegungen wurde die Struktur des Archivs überarbeitet, wobei die daraus folgende Archivierungssystematik eine Bewertung der Quellen als ,nicht eigenständig' implizit vorgibt. Die Ersteller:innen der Codes sind nicht immer vermerkt, aber in der Regel in den Patches gespeichert. Entscheidungen bezüglich Anpassung, Umspeicherung oder Klangveränderung werden nicht notwendigerweise dokumentiert, die nachhaltige Sicherung der Informationen aus den Codes oder Patches ist mit diesem Ansatz jedoch für die archivierten Stücke in gewissem Maß gegeben. Aus pragmatischer Sicht eines Archives, das Möglichkeiten zukünftiger Aufführungen bereithalten möchte, ist dies ein durchaus nachvollziehbarer Ansatz, insbesondere da die rechtliche Lage für die (Original-)Daten damit geklärt ist: zwar bleiben die Urheberrechte bei den Komponist:innen, das Institut behält sich aber die Nutzungsrechte vor und kann somit über neue Aufführungen entscheiden und auch die Entscheidung, Codes neu zu erstellen und/oder weiterzugeben liegt damit bei der Institution. Dies gilt in der Regel auch für die in Sidney abgelegten Stücke, da diese entweder am IRCAM erarbeitet oder unter dessen Verantwortung aufgeführt wurden. Die Nutzung der Quellen wird über einen limitierten Zugriff gesteuert, Außenstehende können nur bedingt darauf zugreifen und die Inhalte werden nicht zwangsläufig an andere Aufführende weitergereicht. Ebenso ist eine Zuführung extern erstellter neuer Codes oder Informationen möglich, dies wird aber (bisher) nicht aktiv forciert.

11 Vgl. Lemouton / Goldszmidt, La préservation des œuvres du répertoire IRCAM. 


\section{Digitales Dokument oder Dokumentation digitaler Inhalte}

Die Erarbeitung einer musikalischen Aufführung ist stark von den zugänglichen Informationen, also den archivierten Inhalten und der Art der Dokumentation, aber auch von Wissen um den (historischen) Kontext abhängig. Die Bibliothekarin Suzanne Briet beschreibt in ihrer bereits 1951 erschienenen Abhandlung "Qu'est-ce que la documentation“ eine Diskussion über die Definition des Konzepts ,Dokumentation' sowie dem archivierten Objekt, dem ,Dokument'. Dabei stellt sie nicht nur verbundene Aufgaben und Herausforderungen dar, sondern präsentiert auch ein Klassifikationssystem, das sie für die Dokumentation entwickelt. Briets Interesse liegt hierbei nicht auf der Wiederaufführung von Musik, sondern dreht sich um die Möglichkeiten, Objekte innerhalb eines Ausstellungskontextes einem Publikum zu präsentieren. Dennoch sind drei der von ihr genannten Aspekte insbesondere im Kontext der Mixed Music sehr interessant: a) die Definition des Inhalts, der als ,Dokument' gilt; b) die Notwendigkeit, sich mit Technologien und geeigneten Techniken auseinanderzusetzen; und c) die Tatsache, dass Briet die Dokumentation bzw. Dokumentieren als eine Kulturtechnik versteht, die in einen sozialen und kulturellen (historisch gewachsenen) Rahmen eingebettet ist. ${ }^{12}$

Für die Mixed Music stellt sich damit die Frage, ob Code als Dokument, oder als Teil eines Dokuments zu sehen ist, und welche Kulturtechniken nötig sind, um einen solchen Inhalt entsprechend zu dokumentieren. Interessant ist hierbei die Frage, was für die Präsentation einer Dokumentation wichtiger ist: dass etwas möglichst originalgetreu dem Publikum vorgestellt wird, oder dass dem Publikum ein Eindruck vermittelt wird, welcher der originalen Präsentation möglichst nahekommt. Gerade für die Mixed Music ist dies ein wichtiger Aspekt, denn eine originalgetreue Klangimpression kann unter Umständen mit komplett abweichender Technologie besser hergestellt werden als mit einem Set-up, das eng an das Original angelehnt zusammengestellt wird.

Dies passt auch zu den Erkenntnissen von Joshua Sternfeld, der mit Blick auf Archivierung in seiner Erklärung zur ,Digital Histography“ herausstellt, dass alle Systeme digitaler Bewahrung den gleichen Mechanismus verwenden, nämlich das Nutzen digitaler Technologien, um Geschichte darstellen zu können. Für Sternfeld sind die Archivierungsprozesse der Schlüssel zur Kontextualisierung der konservierten Objekte, die er als „Einheiten historischer Information“ bezeichnet, da sie bereits einen

12 Vgl. Briet, Qu'est-ce que la documentation?. 
Auswahlprozess durchlaufen haben. ${ }^{13}$ Mit anderen Worten: Die archivierten Informationen spiegeln das Verständnis der Geschichtsschreibung sowie die archivarische Perspektive der Archivverantwortlichen wider. Sternfeld konzentriert sich dabei in seinen Ausführungen nicht nur auf die ursprünglich digitalen Inhalte, sondern auf alle Informationen, die unter Verwendung aktueller digitaler Technologien archiviert werden. Dies impliziert, dass die für die Archivierung verwendete Technologie auch die ursprüngliche Information verändern kann und dass unter Umständen zusätzliche Schritte notwendig sind, um die gesamte archivierte Information auszulesen.

\section{Zugangs- und Zugriffsrechte auf archivierte Quellen}

Stehen in der Betrachtung von Archivierung und ihren zugrundeliegenden Systematiken überwiegend philologische oder kulturgeschichtliche Fragen im Fokus, so bringt die Archivierungspraxis neue Fragen mit sich, die sowohl systematische Herausforderungen wie auch rechtliche Fragestellungen aufwerfen. Grob können diese für die Elektroakustische Musik unter drei Fragenkomplexe subsumiert werden:

\section{a) Durch was wird eine bestimme Komposition charakterisiert/definiert?}

Ist der künstlerische Gedanke charakteristisch für eine Komposition (Komponist:in als Urheber:in der Idee) oder das erklingende Resultat (und damit auch alle daran Mitwirkenden)? Wie werden die Informationen zu den charakterisierenden Merkmalen fixiert bzw. weitergegeben?

Diese Frage ist gerade in der Mixed Music nicht einfach zu beantworten, denn oft sind inhärente Strukturen der eingesetzten Technik ebenso kennzeichnend wie vorhandene Notation. Da bestimmte Aspekte zu einem vergangenen Zeitpunkt oft nicht anders lösbar waren, werden diese in der Regel mangels Alternativen jedoch nicht mehr explizit benannt oder dokumentiert. Dies wird bei der Betrachtung der einzelnen Teile einer Komposition noch deutlicher.

13 Vgl. Sternfeld, The American Archivist, 2011, 544. 


\section{b) Welche Stellung nehmen programmierte Teile innerhalb einer Komposition ein?}

Wie eigenständig sind Codes, Patches oder eingebettete Programme? Sind sie in einer Komposition eher wie ein Notentext einzuordnen, oder sind sie Teil der Instrumentierung? Wie eigenständig ist ein Programmcode?

Zwar ist Programmcode in einer Komposition immer Teil der gesamten künstlerischen Arbeit, es ist jedoch durchaus zu diskutieren, wie eigenständig dieser Teil ist. Manifestiert sich z.B. eine Idee nur oder primär in diesem Teil, so liegt es näher, über eine getrennte Urheberschaft nachzudenken, als wenn es sich um eine reine Umsetzung von Arbeitsaufträgen der Komponist:innen handelt, ein:e Programmierer:in also nur als Ausführende:r im Sinne einer Dienstleistung tätig ist. Dies ist bei einer ausschließlichen Betrachtung der Codes jedoch nicht zu klären. Hinzu kommt, dass es kaum möglich ist, künstlerische Problemstellungen bei der Entwicklung eines Codes ohne eine produktive Eigenleistung zu lösen, da Programmieren, wie Thomas Ernst es im Vergleich mit der Literatur formuliert, auch immer ein ,im Code denken und arbeiten' erfordere. ${ }^{14}$

\section{c) Wie ist die Weitergabe einer Komposition und ibrer einzelnen Bestandteile geregelt?}

Unter welchen Maßgaben können, sollen und vor allem dürfen die Notation einer Komposition oder zugehörige Computerprogramme, die grundlegende Bestandteile einer Komposition bilden, weitergegeben werden? Welche Version eines Programms darf weiterverbreitet werden?

Auch Bearbeitungen von Werken sind urheberrechtlich geschützt. ${ }^{15} \mathrm{Je}$ de:r Bearbeiter:in wäre damit auch Urheber:in; eine Nachnutzung könnte mit den entsprechenden Rechten, z.B. Creative Commons Lizenzen für archivarische und wissenschaftliche Daten gekennzeichnet werden und damit zugänglich bleiben. Die Frage, ob es sich bei jeder neuen Code-Version auch um eine Bearbeitung in diesem Sinne handelt, ist jedoch nicht so leicht zu klären. Es könnte argumentiert werden, eine neue Code-Version sei als eine Art Übersetzungsleistung einzuordnen, die alten (originalen) Code in eine aktuelle ,Sprache` überführt. ${ }^{16}$ Hierbei entstünde mit der

14 Vgl. Respondent Thomas Ernst am 20.2.2020, Weizenbaum Institut, Berlin.

$15 \$ 3$ UrhG.

16 Vgl. Respondent Thomas Ernst am 20.2.2020, Weizenbaum Institut, Berlin. 
Übersetzung eine eigenständige Arbeit mit klarer Referenz an ein Original. Die Übersetzer:innen könnten damit durchaus als Urheber:innen des neuen Code-Textes angesehen werden, die implizite Frage nach der künstlerischen Leistung der Übersetzer:innen ist jedoch weiterhin ungeklärt. Denn welche Rechte erwachsen aus der Übersetzung einer künstlerischen Aussage? Eine dem entgegengesetzte Einordnung wäre es, den Code nur als ein zur Steuerung der Hardware notwendiges Computerprogramm zu klassifizieren. Dies würde die Handhabung der Codes in Archiven erleichtern, denn die Weitergabe von Programmen zu Archivzwecken ist erlaubt. ${ }^{17}$ Auch dies ist aber nicht eindeutig gegeben. Zwar gibt es Stücke in der Mixed Music, in denen Codes rein zur Steuerung beispielsweise von digitalen Synthesizern eingesetzt werden, die dann den Klang berechnen, so ist in den Codes dennoch der eigentliche künstlerische Gehalt, nämlich die vorgegebenen Werte oder Rechenoperationen zur Klangsynthese, hinterlegt. Der Code beinhaltet damit mehr als nur Informationen zur reinen Steuerung. Die Diskussion, so scheint, dreht sich damit einmal mehr im Kreis.

Einen Anker in der Debatte bieten die Archive selbst. Zwar lassen sich in der Archivierung die genannten Herausforderungen wie die Anpassung von Kompositionen an immer neue Technologien und die damit einhergehende, konstant wachsende Ansammlung von Versionen schon länger beobachten. ${ }^{18}$ Und es ist bisher wenig diskutiert, inwiefern Über-, Um- und Einschreibungen im archivierten Material explizit gemacht werden können und inwieweit sich der Blick darauf durch die Fragen nach Urheberschaft und Nutzungsrechten verändert. Die Archivpraxis scheint aber den Herausforderungen (noch?) gelassen entgegen zu sehen. So scheinen in der Forschung zu Mixed Music aktuell vorrangig das Lokalisieren, Sammeln und Pflegen digitaler Quellen untersucht und neue Methoden digitaler Archivierung erarbeitet zu werden. Aspekte, welche die Nachnutzung betreffen, werden kaum angesprochen, scheint es doch ein sekundäres Problem zu sein, solange die Quellen nicht dingfest gemacht werden können. Reflexion und Praxis klaffen hier also sichtbar auseinander.

17 Computerprogramme unterliegen nur dann dem Urheberrecht, wenn sie eine persönliche geistige Schöpfung der Ersteller:innen enthalten. Bestehen die Computerprogramme aus einer einfachen, routinemäßigen Programmierleistung, die von allen Programmierer:innen in ähnlicher Weise erstellt worden wäre, trifft dies nicht zu (vgl. $\$ \$ 2$ Abs. 2, 69a Abs. 3 UrhG, sowie Urteil des BGH, 3. März 2005 -I ZR 111/02 (Fash 2000), Rn. 17.

18 Siehe hierzu u.a. Akkermann, What is saved? 
Verstärkt wird dies durch den nicht oder nur selten geklärten Status der Technologien (Hardware) innerhalb künstlerischer Arbeiten: Entgegen den (publizierten) Partituren und (ggf. publizierten) Ton- und Videoaufnahmen, deren Nutzung z.B. durch die Art der Publikation geklärt werden kann, gibt es für Set-ups, die bei Aufführungen einer Komposition verwendet werden, weder für die Archivierung noch für eine spätere Nutzung/ Weitergabe grundlegende Verfahrensweisen. Dies kann zum einen darauf zurückgeführt werden, dass der Einsatz der Technologien an verschiedenen Positionen innerhalb einer künstlerischen Arbeit erfolgen kann, beispielsweise eher als Instrument beim Einsatz in der Klangsynthese, oder als Effektgerät bei der Berechnung von Klangveränderung. Zum anderen sind bisher in der Mixed Music keine übergreifenden, standardisierten Dokumentationsmethoden zu erkennen. Informationen werden nicht immer institutionell archiviert, da Hardware und Software nicht Teil traditioneller Archivierung sind. Viele der Codes bleiben auf den Speichermedien ihrer Ersteller:innen, eine Weitergabe erfolgt in der Regel oft persönlich, die Erlaubnis zur Nutzung wird zumeist indirekt geklärt und die Überlassung der Codes geht mit der impliziten Erlaubnis zur Nutzung und Änderung einher. Da für Aufführungen die Nutzungsrechte über die Notation geregelt werden können und es sich um keinen Bereich handelt, bei dem größere wirtschaftliche Interessen verfolgt werden, ist Urheberschaft derzeit insbesondere aus der Perspektive der Archivierung interessant. Aber auch für die Analyse von Kompositionen ist eine Zugänglichkeit der Quellen nötig, vor allem da das Überschreiben der Quellen (Codes) durch neue Versionen in der Mixed Music kein seltenes Phänomen ist, denn aktuelle Versionen sind nicht nur leichter handhabbar, liegen komplette Technologie-Generationenwechsel vor, so ist der Original-Code oft nicht mehr (aus)lesbar und damit unabhängig der Rechte unzugänglich. ${ }^{19}$

\section{Fazit}

Die hier dargelegten Herausforderungen sind bisher weder umfänglich diskutiert noch gelöst, auch, da viele dieser Fragen erstmals im letzten Jahrzehnt durch die fortschreitende Digitalisierung auftreten. Sind zum einen vergleichbare Problemstellungen in den (digitalen) Editionen zu finden, die sich neben der Darstellung der Inhalte auch mit Fragen des Vertriebs beschäftigen, so kämpfen zum anderen Archive damit, überhaupt

19 Vgl. Akkermann: What is saved?. 
aktuelle Arbeiten sammeln und zugänglich machen zu können. Ein Blick in benachbarte Disziplinen und deren Umgang mit ähnlichen Fragen ist hierbei mehr als hilfreich. Die größte Hürde für die Elektroakustische Musik ist derzeit der Erhalt der Kompatibilität: Archive sind implizit aufgrund des technischen Fortschritts gezwungen, immer neue und eventuell stark vom Original abweichende Versionen zu erstellen, um die Inhalte (u.a. die Codes) überhaupt zugänglich zu erhalten. Im Zuge dessen sollte dann auch diskutiert werden, wer Veränderung (an den Codes) vornehmen darf und wie die daraus folgenden Versionen archiviert werden. Dies ist ohne die Frage nach der Urheberschaft sicher nicht nachhaltig zu klären.

\section{Literatur}

Akkermann, Miriam: What is saved?, Proceedings of the International Computer Music Conference ICMC, New York 2019, doi: 10.5281/zenodo.3484546.

Berweck, Sebastian: It worked yesterday: On (re-)performing electroacoustic music,

Doctoral thesis, Huddersfield 2012.

Briet, Susan, Qu'est-ce que la documentation?, Paris 1951.

Lemouton, Serge/Goldszmidt, Samuel: La préservation des œuvres du répertoire IR-

CAM: Présentation du modèle Sidney et analyse des dispositifs temps réel, Journées d'Informatique musicale, 2016, hal-01944619.

Sternfeld, Joshua: Archival Theory and Digital Histography: Selection, Search, and Metadata as Archival Process for Assessing Historical Contextualization, The American Archivist, 74 (3), 2011, S. 544-575.

Supper, Martin, Elektroakustische Musik und Computermusik, Darmstadt 1997.

Ders.: Art. Computermusik, in: Lütteken, Laurenz (Hrsg.), MGG Online, Kassel/ Stuttgart/New York 2016ff., zuerst veröffentlicht 1995, https://www.mgg-online. $\mathrm{com} / \mathrm{mgg} / \mathrm{stable} / 12446$ (zuletzt abgerufen am 15.5.2020).

Supper, Martin/Ungeheuer, Elena: Art. Elektroakustische Musik, in: Lütteken, Laurenz (Hrsg.), MGG Online, Kassel/Stuttgart/New York 2016ff., zuerst veröffentlicht 1995, https://www.mgg-online.com/mgg/stable/11329 (zuletzt abgerufen am 15.5.2020).

Tiffon, Vicent: Espace et musique mixte, Ars Sonora 5, 1997, http://www.ars-sonora. org/html/numeros/ numero05/05b.htm (zuletzt abgerufen am 16.1.2020). 